\title{
The 1998 Miyako fireball's trajectory determined from shock wave records of a dense seismic array
}

\author{
Yoshiaki Ishihara $^{1}$, Shin'ya Tsukada $^{2}$, Shin'ichi Sakai $^{3}$, Yoshihiro Hiramatsu $^{1}$, and Muneyoshi Furumoto ${ }^{1}$ \\ ${ }^{1}$ Graduate School of Natural Science and Technology, Kanazawa University, Kakuma, Kanazawa, Ishikawa 920-1192, Japan \\ ${ }^{2}$ Earthquake Prediction Information Division, Seismological and Volcanological Department of Japan Meteorological \\ Agency, 1-3-4 Otemachi, Chiyoda, Tokyo 100-8122, Japan \\ ${ }^{3}$ Earthquake Observation Center, Earthquake Research Institute, University of Tokyo, 1-1-1 Yayoi, \\ Bunkyo, Tokyo 113-0032, Japan
}

(Received April 2, 2003; Revised April 29, 2003; Accepted April 30, 2003)

\begin{abstract}
A high velocity passage of a meteoroid through the atmosphere generates a shock wave with a conical front. When the shock front arrives at the surface, it causes high frequency ground motions that are registered on the seismograms. We can use seismological data to determine the trajectory of the meteoroid in the atmosphere. A strong shock wave from the 1998 Miyako fireball is recorded by more than 20 stations in a dense array of seismographs installed in the northeastern region of Honshu Island, Japan. We determine the velocity and the trajectory of the fireball in the upper atmosphere using the arrival times of the shock wave at the stations.
\end{abstract}

Key words: Shockwave, fireball, trajectory, seismic array.

\section{Introduction}

Meteoroid impacts into the Earth's atmosphere have been studied using various instruments. Ground-based camera video recorders networks have been widely used to observe fireballs and bolides (Halliday et al., 1996). Recently, satellite-based optical instruments demonstrate their ability to register bolides in the atmosphere (e.g., Brown et al., 2002). Barometer or infrasonic microphone arrays deployed on the surface are also effective systems to identify large bolides in the atmosphere (McIntosh et al., 1976; ReVelle, 1976, 1997).

Another method to study features of fireballs and bolides is a seismological approach. Meteoroids with super-sonic velocities and bolides generate shock waves in the atmosphere. If the shock waves are sufficiently strong, they generate impulse ground motions that can be recorded by seismographs on the ground (Nagasawa, 1978; Nagasawa and Miura, 1987; Cevolani, 1994). Therefore, a dense array of seismographs installed for a purpose of monitoring earthquake activities is also a good apparatus for the fireball observations. Kanamori and his colleagues have demonstrated the ability of the seismic records to determine the trajectory of high velocity objects, space shuttles (Kanamori et al., 1991; Mori et al., 1991).

The bright 1998 Miyako fireball flew over a dense seismographic array deployed in the Tohoku region in Japan. The shock wave signals are clearly recorded on many seismograms of the array. The data provide a good opportunity to show a potentiality of a seismic array as a fireball observation system. In this page we report the determination of trajectory and velocity of the fireball by the analysis of the seismograms obtained by the array.

Copy right(C) The Society of Geomagnetism and Earth, Planetary and Space Sciences (SGEPSS); The Seismological Society of Japan; The Volcanological Society of Japan; (SGEPSS); The Seismological Society of Japan; The Volcanological Society of
The Geodetic Society of Japan; The Japanese Society for Planetary Sciences.

\section{The Miyako Fireball and a Seismic Array}

The bright Miyako fireball appeared at 3:20 am (the local time JST $=\mathrm{UT}+9 \mathrm{~h}$ ) on March 30, 1998 in the northeastern region of the Honshu Island of Japan. The fireball was observed by an amateur astronomer's fireball patrol camera network. The visual magnitude was estimated to be -14 from the photograph records and the trajectory parameters were obtained by an analysis of two photos (Japan Fireball Network, 1998). It traversed across northeast Honshu Island from WSW to ENE with a velocity of about $19 \mathrm{~km} / \mathrm{sec}$. No meteorite related to the fireball has been recovered. The meteoroid likely fell onto the Pacific Ocean off the east cost of the region.

The fireball crossed the sky just above a dense seismic array, which was temporary installed by a consortium of seismological researchers from Japanese universities (the 1997-98 joint seismic observation in the Tohoku Backbone Range). Including permanent seismic stations that were installed by Tohoku University and the Japan Meteorological Agency, the array comprises 85 stations in an area of $4.0 \times$ $104 \mathrm{~km}^{2}$ (Fig. 1). The main purpose of the seismic observation is to determine the source locations of microearthquakes in the region. The seismographs are very sensitive to the ground motions and their clocks are precisely maintained $\left(10^{-6} \mathrm{sec}\right)$ using GPS signals. The seismographs are deployed at selected locations where the background noise of the Earth, microtremors, is small. Almost all stations are installed on the surface and are sensitive to ground surface motions generated by a shock wave from the atmosphere.

\section{Data}

We inspect the seismic array data around the time of the fireball appearance. A shock wave arrival is easily noticed on a velocity seismogram by its characteristic waveform. 


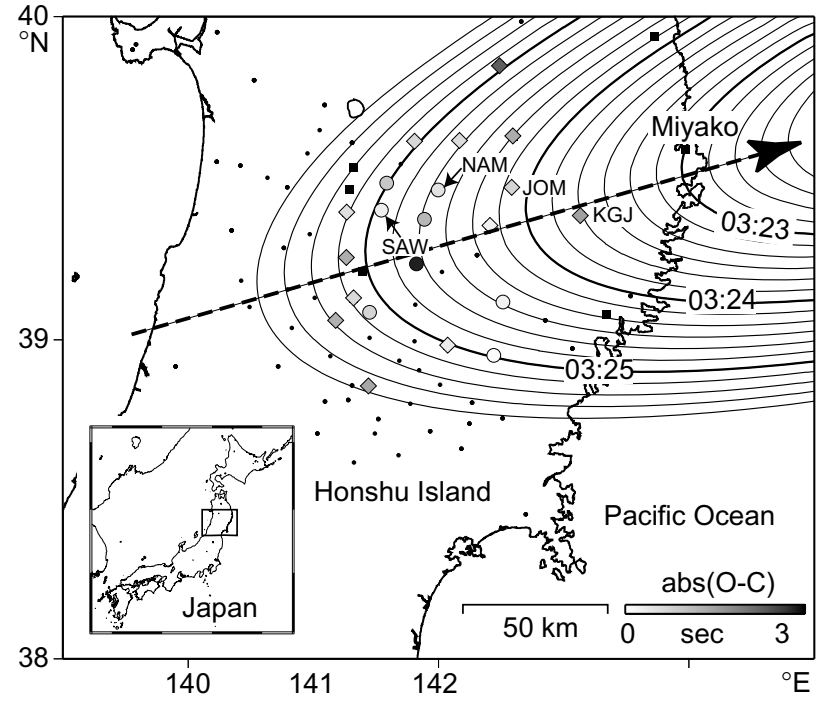

Fig. 1. Calculated isochronal lines of the shock wave arrivals. The arrow indicates the determined trajectory of the Miyako fireball. Diamonds are seismic stations at which observed arrival times are faster than calculated ones. Circles are seismic stations at which recorded observed arrival times are slower than calculated ones. The gray scale indicates the absolute value of the residual, the observed time minus the calculate time. Squares are seismic stations at which we observe unclear shock wave and are not used in the analysis. Dots are seismic stations which we observe no shock wave signals.

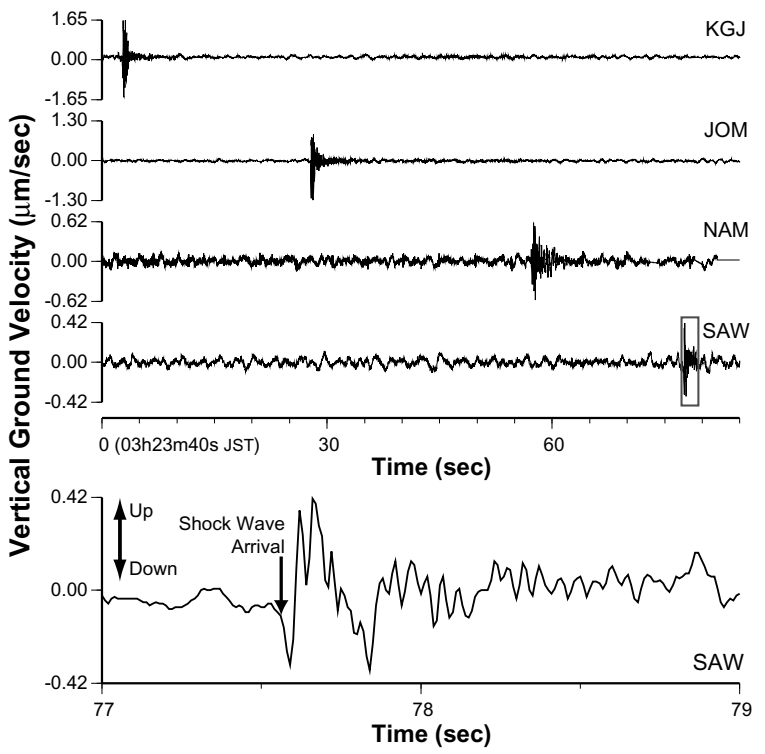

Fig. 2. Examples of shock waves, recorded at seismic stations, KGJ $\left(39.3865^{\circ} \mathrm{N}, 141.5659^{\circ} \mathrm{E}, 375 \mathrm{~m}\right), \mathrm{JOM}\left(39.4730^{\circ} \mathrm{N}, 141.2923^{\circ} \mathrm{E}\right.$ $210 \mathrm{~m}), \mathrm{NAM}\left(39.4678^{\circ} \mathrm{N}, 140.9979^{\circ} \mathrm{E}, 245 \mathrm{~m}\right)$ and SAW $\left(39.4030^{\circ} \mathrm{N}\right.$, $\left.140.7720^{\circ} \mathrm{E}, 280 \mathrm{~m}\right)$. The lowermost seismogram shows the enlarged view of the part of shock wave signal at the SAW station. Note that the velocity seismogram of the vertical ground motion shows a N-shaped waveform. The negative represents downward motion. The shock wave signal necessarily begins with a downward motion. The reference time is $03 \mathrm{~h} 23 \mathrm{~m} 40$ s JST.

The shock wave generated by a meteoroid is a compressional shock wave followed by a rarefactional shock wave. The compressional and rarefactional shock waves cause the rapid downward and upward ground motions, respectively. These successive ground motions are recorded as a shape of
Table 1. List of seismic stations that detected shock wave signal.

\begin{tabular}{|c|c|c|c|c|}
\hline Station & Latitude $\left[{ }^{\circ}\right]$ & Longitude $\left[{ }^{\circ}\right]$ & Height $[\mathrm{m}]$ & Arrival Time [h:m:s] \\
\hline MYK & 39.590 & 141.98 & 120 & 03:23:08.27 \\
\hline KGJ* & 39.387 & 141.57 & 375 & $03: 23: 42.74$ \\
\hline $\mathrm{JOM}^{*}$ & 39.473 & 141.29 & 210 & $03: 24: 07.83$ \\
\hline MNS* & 39.355 & 141.20 & 65 & $03: 24: 13.78$ \\
\hline JOF & 39.080 & 141.67 & 180 & $03: 24: 19.71$ \\
\hline JTH & 39.939 & 141.86 & 200 & 03:24:21.96 \\
\hline NTM* & 39.632 & 141.30 & 311 & $03: 24: 22.06$ \\
\hline THR* & 39.118 & 141.26 & 165 & $03: 24: 28.93$ \\
\hline NAM* & 39.466 & 141.00 & 245 & $03: 24: 37.34$ \\
\hline $\mathrm{HAN}^{*}$ & 39.374 & 140.94 & 300 & $03: 24: 38.35$ \\
\hline GTO* & 39.237 & 140.91 & 610 & $03: 24: 41.29$ \\
\hline YHB* & 39.618 & 141.08 & 300 & $03: 24: 43.85$ \\
\hline SAW* & 39.403 & 140.77 & 280 & $03: 24: 57.55$ \\
\hline $\mathrm{JMK}^{*}$ & 38.952 & 141.22 & 70 & 03:24:59.12 \\
\hline SWU* & 39.486 & 140.79 & 445 & 03:25:01.14 \\
\hline HRQ* & 38.984 & 141.04 & 123 & 03:25:01.50 \\
\hline WNS & 39.278 & 140.82 & 280 & $03: 25: 02.32$ \\
\hline OSK $^{*}$ & 39.616 & 140.90 & 270 & 03:25:03.98 \\
\hline $\mathrm{HMK}^{*}$ & 39.848 & 141.24 & 650 & 03:25:06.76 \\
\hline KT44* & 39.086 & 140.72 & 400 & 03:25:07.38 \\
\hline SNN & 39.214 & 140.70 & 270 & 03:25:08.01 \\
\hline $\mathrm{HRN}^{*}$ & 39.256 & 140.63 & 170 & 03:25:08.64 \\
\hline KT43* & 39.130 & 140.66 & 280 & 03:25:09.76 \\
\hline JRG* & 39.396 & 140.63 & 200 & 03:25:12.09 \\
\hline NNW & 39.466 & 140.64 & 170 & $03: 25: 16.46$ \\
\hline KT48* & 39.061 & 140.59 & 235 & $03: 25: 20.42$ \\
\hline NKS & 39.536 & 140.66 & 170 & $03: 25: 22.15$ \\
\hline GNY* & 38.857 & 140.72 & 440 & 03:25:39.11 \\
\hline
\end{tabular}

*21 stations used for the analysis.

a reversed $\mathrm{N}$ on a velocity seismogram (Fig. 2). We identify shock wave signals from the fireball at 28 seismic stations (Table 1). At the other stations in the array, the shock wave signals are thought to be smaller than the background noises. The maximum observed ground velocity induced by the shock wave is $1.65 \times 10^{-6} \mathrm{~m} / \mathrm{sec}$. We use here shock wave arrival times of 21 stations which record clear onsets of the signals. The arrival times of the shock wave are picked out with an accuracy of 0.1 seconds or less.

\section{Method}

To determine the trajectory, we make two approximations (1) that the fireball linearly penetrates through the atmosphere with a constant velocity $v$, and (2) that the shock wave speed $c$ is uniform in the atmosphere and to be $320 \mathrm{~m} / \mathrm{sec}$. The first approximation is based on observations that meteoroids have nearly constant velocities in the upper atmosphere (e.g., Brown et al., 2002; Halliday et al., 1981). The second approximation is adopted because of small incident angles of the shock waves analyzed in this study. In the real atmosphere below a height of about $100 \mathrm{~km}$, the shock wave speed may change in a range of $290 \mathrm{~m} / \mathrm{sec}$ to $340 \mathrm{~m} / \mathrm{sec}$. However, the speed varies with the height. If the shock wave is propagated nearly vertically, the ray is hardly refracted and an average velocity can be used to calculate a travel time. 
Table 2. Search domain, grid interval and optimum value of each parameter*.

\begin{tabular}{ccccc}
\hline Parameter & $\begin{array}{c}\text { Search } \\
\text { Domain }\end{array}$ & $\begin{array}{c}\text { Grid } \\
\text { Interval }\end{array}$ & $\begin{array}{c}\text { Optimum Value and } \\
\text { Confidence Interval }\end{array}$ & $\begin{array}{c}\text { Photo Analysis } \\
\text { Value }\end{array}$ \\
\hline$v[\mathrm{~km} / \mathrm{sec}]$ & $11 \sim 45$ & 1.0 & $18+7 /-3$ & 18.6 \\
$x_{0}[\mathrm{~km}]$ & $-110 \sim-50$ & 1.0 & $-93+3 /-3$ & - \\
$y_{0}[\mathrm{~km}]$ & $180 \sim 250$ & 1.0 & $207+3 /-2$ & - \\
$\gamma\left[^{\circ}\right]$ & $270 \sim 300$ & 0.5 & $287+1 /-1$ & 296 \\
$\theta\left[^{\circ}\right]$ & $10 \sim 40$ & 0.5 & $18.5+0.5 /-0.5$ & 18.3 \\
$t_{0}[\mathrm{sec}]$ & $0 \sim 80$ & 1.0 & $48+3 /-3$ & - \\
\hline
\end{tabular}

*The origin is $\left(39^{\circ} \mathrm{N}, 141^{\circ} \mathrm{E}, 0\right)$ at $03 \mathrm{~h} 20 \mathrm{~m} 00 \mathrm{~s} \mathrm{JST}(\mathrm{UT}+9 \mathrm{~h})$.

Since the incident angles of the shock waves to almost all the stations used here are smaller than 30 degree, the errors caused by the second approximation are small compared with other practical uncertainties (Tatum, 1999).

For the case of a linear-motion of a meteoroid with a constant velocity, the generated shock wave front forms a cone; the isochronal curves of the shock wave arrival times on a flat surface are conic sections. While the meteoroid moves with a super-sonic speed (about $20 \mathrm{~km} / \mathrm{sec}$ ), the velocity of the shock wave is low, $320 \mathrm{~m} / \mathrm{sec}$. Then the semi-vertical angle of the shock wave cone is very small (about 1 degree). This angle is generally smaller than the angle of elevation of the meteoroid trajectory. Therefore the isochronal curves of arrival times on the surface are half ellipses in most cases.

We define two rectangular coordinate systems, $(x, y, z)$ and $(X, Y, Z)$ (Fig. 3). The origin of the coordinate $(x, y, z)$ is given at a point on the Earth's surface. In this study the origin is at a point $39^{\circ} \mathrm{N}$ and $141^{\circ} \mathrm{E}$. The $x-y$ plane is a tangent plane of the Earth's surface at the origin. The $x, y$, and $z$ axes point southward, eastward, and upward, respectively. The second set of coordinates $(X, Y, Z)$ is introduced to describe the shock wave cone. Its origin is located at a point where the meteoroid trajectory intersects the $x-y$ plane. The $Z$ axis points in the direction of the approaching meteoroid. The $X$ axis is on a vertical plane containing the trajectory. The $Y$ axis is on the $x-y$ plane and perpendicular to the $X$ and $Z$ axes. In such coordinate systems, the shock wave front and the trajectory of the fireball can be expressed as follows (Nagasawa and Miura, 1987).

$$
\begin{gathered}
\frac{\sqrt{X^{2}+Y^{2}}}{\tan \beta}-Z=v\left(t-t_{0}\right) \\
\sin \beta=c / v \\
\left(\begin{array}{l}
X \\
Y \\
Z
\end{array}\right)=\left(\begin{array}{ccc}
\cos \gamma \sin \theta & \sin \gamma \sin \theta & -\cos \theta \\
-\sin \gamma & \cos \gamma & 0 \\
\cos \gamma \cos \theta & \sin \gamma \cos \theta & \sin \theta
\end{array}\right)\left(\begin{array}{c}
x-x_{0} \\
y-y_{0} \\
z
\end{array}\right),
\end{gathered}
$$

where, $v$ is the velocity of the meteoroid, $\gamma$ is the azimuth of the trajectory (counterclockwise from the $x$-axis), $\theta$ is the elevation angle of the trajectory from the $x-y$ plane, the point $\left(x_{0}, y_{0}, 0\right)$ is the intercept of the trajectory to the $x-y$ plane at an expected time $t_{0}$. The stations locations are converted form the geographic coordinates (the latitude, the longitude, the height) to the $x, y$, and $z$ coordinates.

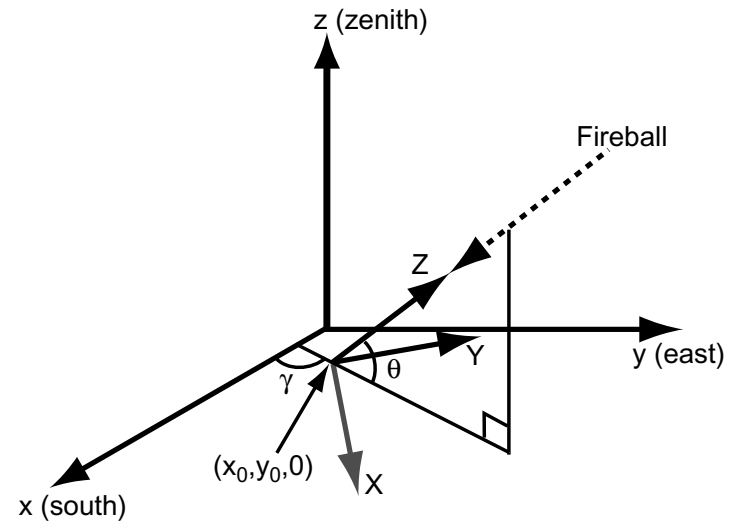

Fig. 3. Coordinate systems for the fireball trajectory used in the analysis.

Given a set of six parameters of the trajectory, $v, \gamma, \theta$, $x_{0}, y_{0}, t_{0}$, we can calculate the expected arrival time $t_{i}$ at the $i$-th station whose coordinates are $\left(x_{i}, y_{i}, z_{i}\right)$. Let $\tau_{i}$ be the observed arrival time at the $i$-th station. The best set of the parameters can be retrieved by minimizing the sum of squared residuals $\left(t_{i}-\tau_{i}\right)^{2}$. However since there are many local minima of the sum in the six parameters space, the solution estimated by an inversion method easily falls into such local minima near the initial values. In order to find the best set of the parameters in the relevant space, we use a grid search method. The search ranges and the grid intervals of the individual parameters are summarized in Table 2. The $68.3 \%$ confidence interval of each parameter is estimated using the $\chi^{2}$ test of the sum of the squared residuals.

\section{Results and Discussion}

The best six parameters for the trajectory and their confidence intervals are listed in Table 1. Parameters given by an analysis of photographic data (Japan Fireball Network, 1998) are also listed in Table 2. The trajectories determined by both the methods agree well with each other.

In Fig. 1 the projection of the fireball trajectory to the surface is drawn, with calculated isochrones of the shock wave arrivals. As shown in the figure, there is good the azimuthal coverage of the stations to determine the fireball trajectory. The calculated arrival times of the shock waves at the individual stations are consistent with the observed ones. The root mean square $\sigma$ of the differences between the observed and calculated arrival times is 1.1 seconds. The semi-vertical angle of the shock front cone is smaller $\left(\beta \approx 1^{\circ}\right)$ than the incident angle $\left(\theta=18.5^{\circ}\right)$. Therefore, the isochrones of the shock wave arrivals are half ellipses (Fig. 1). The arrivals of the shock wave begin at eastern stations, which are near the end point of the fireball trajectory, and retrograde westwards (Fig. 2). Such retrogression of the wave arrival is also caused by the slow shock wave velocity. The longest travel times that are observed at western stations are about $290 \mathrm{sec}$. These values correspond to travel distances of about $90 \mathrm{~km}$. This means that the shock wave generation started at least from a height of more than $80 \mathrm{~km}$, which coincides well with the reported height $(86 \mathrm{~km})$ at which the fireball started to emit light (Japan Fireball Network, 1998).

In this analysis we assume that the shock wave velocity is 
uniform from the surface to the height of the fireball. However, the real atmosphere is apparently much more complex and there are spatial variations of the velocity with different spatial scales. The arrival time residuals whose r.m.s. $\sigma=1.1 \mathrm{sec}$, reflect such variations. Random components in the velocity variations do not greatly affect the estimation of the trajectory, since their effects averaged together by using many stations. However, there may exist systematic variations of the shock wave velocity that are neglected in the estimation. One potential source of error is the difference between the assumed and the real average velocities of shock wave. We adopt the average velocity of $320 \mathrm{~m} / \mathrm{sec}$ in the analysis. The difference can be $20 \mathrm{~m} / \mathrm{sec}$ or about $6 \%$ at most. The maximum difference may cause a discrepancy of a few $\mathrm{km}$ in the height and horizontal location between the real and the estimated trajectories.

Another important factor that is neglected in the analysis is the wind in the lower atmosphere. In particular the strong westerly wind, the jet stream, blows over the relevant latitudinal zone in the winter season. The shock waves are driven out of their travel paths that are expected for a still atmosphere. If the average westerly wind speed in the lower atmosphere with a representative thickness of $20 \mathrm{~km}$ is 20 $\mathrm{m} / \mathrm{sec}$ at most, the eastward driven distance of the paths is about one $\mathrm{km}$. This is within a range of the error for the trajectory estimation.

The results obtained in this analysis show that a dense seismic array is a useful system to observe fireballs. At present, there are about one thousand seismic stations in the Japanese islands, which extends for a length of about $2500 \mathrm{~km}$. Many seismographs are installed on the ground or near the surface, and would be sensitive to the shock wave signals from fireballs. Data from the stations can be immediately transmitted to any laboratories in Japan through a communication satellite, enabling analyses of shock wave signals in real time. The seismic array can watch for fireballs 24 hours a day even through cloudy or rainy skies. It is expected that the seismic array can provide valuable data on fireball phenomena and the meteoroid flux rate.

\section{Conclusions}

We find clear shock waves records from the 1998 Miyako fireball in seismograms of a dense seismic array in the northeastern Japan. We determine the trajectory of the fireball in the atmosphere from the arrival times of shock waves. In the calculation we assume that the fireball traverses linearly with constant velocity and a uniform shock wave speed of $320 \mathrm{~m} / \mathrm{sec}$ in the atmosphere. The optimum values of the tra- jectory parameters are the meteoroid velocity of $18 \mathrm{~km} / \mathrm{sec}$, the azimuth of the trajectory of $287^{\circ}$ the incident angle of trajectory of $18.5^{\circ}$. We recommend a dense seismic array as a useful tool to observe fireball because of its independence of weather conditions and its potential for successful observations.

Acknowledgments. We wish to thank J. Mori for his critical reading of the manuscript and comments. We are also grateful to anonymous referees for constructive comments and correction in English, which led us to improve the manuscript. The seismic data used in this study are provided by the consortium of the 1997-98 joint seismic observations in the Tohoku Backbone Range. Generic Mapping Tools (Wessel and Smith, 1998) was used to draw Fig. 1 and Fig. 2. We thank Y. Kamikawa for his financial support.

\section{References}

Brown, P., D. O. ReVelle, E. Tagliaferri, and A. R. Hildebrand, An entry model for the Tagish Lake fireball using seismic, satellite and infrasound records, Meteorit. Planet. Sci., 37, 661-675, 2002.

Cevolani, G., The explosion of the bolide over Lugo di Romagna (Italy) on 19 January 1993, Planet. Space Sci., 42, 767-775, 1994.

Halliday I., A. A. Griffin, and A. T. Blackwell, The Innisfree Meteorite fall: A photographic analysis of fragmentation, dynamics and luminosity, Meteoritics, 16, 153-170, 1981.

Halliday, I., A. A. Griffin, and A. T. Blackwell, Detailed data for 259 fireballs from the Canadian camera network and inferences concerning the influx of large meteoroids, Meteoritics, 31, 185-217, 1996.

Japan Fireball Network, JN980329a Orbit, http://www2 . cnet.ne. jp/c-shimo/JN980329aOrbit.html, 1998.

Kanamori, H., J. Mori, D. L. Anderson, and T. Heaton, Seismic excitation by the space shuttle Columbia, Nature, 349, 781-782, 1991.

McIntosh, B. A., M. D. Watson, D. O. ReVelle, Infrasound from a radarobserved meteor, Can. J. Phys., 54, 655-662, 1976.

Mori, J. and H. Kanamori, Estimating Trajectory of Supersonic Objects using arrival Times of Sonic Booms, U. S. Geol. Survey Open-File Rept., 91-48, 1991

Nagasawa, K., An analysis of sonic boom from a Great Fireball on May 10, 1977, recorded on seismographs of volcano observations, Bull. Earthq. Res. Inst. Univ. Tokyo, 53, 271-280, 1978 (in Japanese with English abstract).

Nagasawa, K. and K. Miura, Aerial Path Determination of a Great Fireball from Sonic Boom Records on Seismographs, Bull. Earthq. Res. Inst. Univ. Tokyo, 62, 579-588, 1987 (in Japanese with English abstract).

ReVelle, D. O., On meteor-generated infrasound, J. Geophys. Res., 81, 1217-1230, 1976.

ReVelle, D. O., Historical detection of atmospheric impacts by large bolides using acoustic-gravity waves, Ann. NY Acad. Sci., 822, 284-302, 1997.

Tatum, J. B., Fireballs: Interpretation of airblast data, Meteorit. Planet. Sci., 34, 571-585, 1999.

Wessel, P. and W. H. G. Smith, New improved version of the Generic Mapping Tools released, EOS Trans. AGU, 79, 579, 1998.

Y. Ishihara (e-mail: ishihara@hakusan.s.kanazawa-u.ac.jp), S. Tsukada, S. Sakai, Y. Hiramatsu, and M. Furumoto 